\title{
Lesiones naturales y regeneración de tejido en ramets del coral Montastraea annularis (Scleractinia: Faviidae) en un arrecife degradado del Caribe Colombiano
}

\author{
Elvira Alvarado Ch. ${ }^{1} \&$ Alberto Acosta ${ }^{2}$ \\ 1. Facultad de Ciencias Naturales, Programa Biología Marina, Universidad Jorge Tadeo Lozano, Bogotá, Colombia; \\ elvira.alvarado@utadeo.edu.co \\ 2. Facultad de Ciencias, Departamento de Biología, UNESIS, Pontificia Universidad Javeriana, Bogotá, Colombia; \\ laacosta@javeriana.edu.co
}

Recibido 04-VI-2008. Corregido 20-IV-2009. Aceptado 20-V-2009.

\begin{abstract}
Natural lesions and regeneration of ramets of Montastraea annularis (Scleractinia: Faviidae) in a degraded reef of the Colombian Caribbean. Currently, Montastraea annularis populations are suffering from high partial mortality rates; yet few studies have determined type, frequency and intensity of natural lesions in situ. During a year we followed natural lesions that appeared on healthy ramets of a population on a degraded reef within a Marine Protected Area in Colombia, to infer the effect on population size structure. From September 2003 to September 2004, 94\% of the ramets presented lesions caused by bleaching, predation; or algae, sponge and borrower interactions. Predation caused $47 \%$ of the lesions and algae $36 \%$; most lesions $(85 \%)$ were small $\left(<5 \mathrm{~cm}^{2}\right)$, although some were bigger than $60 \mathrm{~cm}^{2}(0.3 \%)$. Lesion recovery was high $(83 \%)$, but $11.2 \%$ did not recover or grow. On $2.5 \%$ algae invasion was seen. Bleaching lesions affected more ramet area ( $>60 \%$ of its live tissue) and were evident from September to November, yet total recovery was found in three months. In contrast, lesions by algae (36\%), showed less recovery $(6.7 \%)$, and a tendency to grow in time. In general, percentage of affected tissue area of a ramet in any month was lower than $10 \%$. Nevertheless from May to September, the area affected was larger (10-50\%) due to an increase in frequency and abundance of predation, bleaching and algal damage, and a decrease in recovery. At the end of the year, lesions that did not recover caused partial mortality in $25 \%$ of the ramets. Ramets with lesions shrunk throughout the year and by the end of year, $21 \%$ passed to a smaller class size. Rev. Biol. Trop. 57 (4): 939-954. Epub 2009 December 01.
\end{abstract}

Key words: natural lesions, recovery, bleaching, predation, Montastraea annularis, partial mortality, size-class structure.

El aumento en la intensidad y frecuencia de amenazas tanto de origen natural como antropogénico son factores causales de los cambios en el estatus de los arrecifes que se han venido presentando en las últimas tres décadas (Connell et al. 1997, Gardner et al. 2003). Este deterioro en los arrecifes se manifiesta en la pérdida de cobertura de tejido vivo y en la mortalidad parcial y total de corales, especialmente de aquellas especies que se encuentran en los arrecifes someros (Gardner et al. 2003). Estudios de poblaciones de especies de coral realizados en el Caribe reportan que los disturbios agudos de alta intensidad tienen grandes efectos sobre su estructura, dada la pérdida de individuos por mortalidad total y de cobertura de tejido vivo por mortalidad parcial causada por lesiones que no se recuperan (Edmunds 2002).

La remoción de tejido o esqueleto que genera mortalidad parcial en las colonias de coral se ha considerado consecuencia de una lesión creada por agentes físicos y biológicos tales como tormentas, sedimentación, epidemias, altas temperaturas, competencia por espacio, actividades humanas y contaminación (Rogers et al. 1982, Bythell et al. 2000, Fong 
\& Glynn 2000). También se ha sugerido que la constante mordedura de peces (Bythell et al. 1993, Miller \& Hay 1998) y la rápida colonización de espacios libres o el sobrecrecimiento de macroalgas (McCook et al. 2001, Mumby et al. 2005) o esponjas (López-Victoria \& Zea 2005) son causas de pérdida de cobertura de tejido vivo de coral. A pesar de que el blanqueamiento no se ha considerado como lesión en el sentido de remover tejido o esqueleto, este causa desorden de las funciones vitales en órganos, sistemas o funciones corporales globales (Peters et al. 1997). El blanqueamiento es una reacción del coral a la ausencia o la disminución de los suplementos de nutrientes, la elevación en la temperatura del agua, la sedimentación y turbidez, lo cual lleva a la expulsión de las zooxantelas de las células de la gastrodermis del coral, la degradación de las zooxantelas de las células in situ o una combinación de ambas (Hayes \& Bush 1990, Rowan et al. 1997, Weil 2004, Garren et al. 2006).

Por su organización modular, las colonias de corales pueden recuperar el tejido dañado (Meesters et al. 1997, Oren et al. 1997, Lirman 2000). La recuperación de una lesión se expresa en términos de reducción del tamaño de la lesión (Meesters et al. 1994, Oren et al. 2001) siendo mayor la tasa de recuperación cuando las colonias están saludables (Mascarelli \& Bunkley-Williams 1999). Estudios relacionados con la recuperación de lesiones (artificiales y naturales) muestran que el tamaño, forma y posición de la lesión, el tamaño de la colonia con relación a la lesión, el estado reproductivo, las condiciones fisicoquímicas del agua, la disponibilidad de nutrientes, la sedimentación y la depredación, entre otros, influyen en el proceso de recuperación (Bythell et al. 1993, Guzmán et al. 1994, Van Veghel \& Kahman 1994, Hall 1997, Lewis 1997, Ruesink 1997, Fong \& Glynn 1998, Kramarsky-Winter \& Loya 2000, Oren et al. 2001, Cróquer et al. 2002, Mumby et al. 2005, 2007). En estos se resalta que las colonias de coral experimentan disturbios frecuentes a pequeña escala que resultan en lesiones que se recuperan, lo cual según Mumby et al. (2005) sugiere un proceso de rutina que demuestra una eficiente estrategia de supervivencia colonial. No obstante implica un costo que conlleva a cambios en el metabolismo (Fabricius 1995) con lo cual se altera el mantenimiento, la reproducción y crecimiento del individuo.

Montastraea annularis (Ellis \& Solander 1786) se reconoce como uno de los más importantes y abundantes corales constructores de arrecifes de la región Caribe (Weil \& Knowlton 1994, Hughes \& Tanner 2000). No obstante actualmente es una de las especies que tiene mayor cobertura de tejido muerto en las áreas coralinas del Caribe colombiano (Garzón-Ferreira et al. 2005) y en el Caribe (Ginsburg et al. 2001), lo cual es preocupante dado su papel en la formación de arrecifes (Pandolfi et al. 2002). En los últimos años se ha registrado aumento en la mortalidad en colonias de esta especie causadas por enfermedades (Garzón-Ferreira et al. 2001, Miller et al. 2003, Sutherland et al. 2004) así como por la interacción con algas en especial Dictyota spp., Halimeda spp. y Lobophora variegata (Edmunds 2002, Mumby et al. 2005). Un estudio reciente indica que el declinamiento en la cobertura de $M$. annularis es el resultado de mortalidad en ramets (individuos de un clon o colonia) de tallas grandes que se fisionan y las altas tasas de mortalidad en los ramets de tallas más pequeñas (Edmunds \& Elahi 2007). Con la evidencia del deterioro de las poblaciones de $M$. annularis en gran parte del Caribe, el presente estudio tuvo como objetivo evaluar el tipo de lesión natural, la frecuencia en que se presenta determinado tipo de lesión, el tamaño de las lesiones y la tasa de recuperación de tejido afectado que se presentó en ramets sanos de una población de esta especie ubicada en un arrecife degradado durante un año, con el fin de inferir el efecto sobre la estructura de talla.

\section{MATERIALES Y MÉTODOS}

Área de Estudio: La zona de estudio se encuentra en el Archipiélago de Nuestra Señora del Rosario ( $10^{\circ} 7^{\prime}-10^{\circ} 14^{\prime} \mathrm{N}$; 7537' $-75^{\circ} 52^{\prime} \mathrm{W}$ ) dentro de un Área Marina Protegida del Caribe 
Colombiano. El clima presenta un período seco mayor entre diciembre y abril dominado por los vientos Alisios de nor-este, un período lluvioso menor de mayo a junio, un período seco menor de julio a agosto, y uno lluvioso mayor entre agosto y noviembre en el que dominan los vientos del sur-occidente (Pujos et al. 1984). La temperatura superficial del agua es mayor en época de lluvias $\left(30^{\circ} \mathrm{C}\right)$ que en época seca $\left(26.1^{\circ} \mathrm{C}\right.$; Alvarado et al. 1992). Durante los meses en que se llevó a cabo este estudio, la temperatura superficial del agua estuvo en rangos considerados como normales por la NOAA (0.2-0.5 ONI, Oceanic Niño Index, http:// ggweather.com/enso/oni.htm), con excepción de los meses de octubre a diciembre del 2003 y de julio, agosto y septiembre de 2004 que estuvieron por encima del límite de los $0.5^{\circ} \mathrm{C}$ (hasta 1.5 por encima del rango en el 2003 y de 0.9 ONI en el 2004). La salinidad del agua superficial presenta valores de $35 \%$ con registros más bajos en época lluviosa (30\%; Alvarado et al. 1992). La zona está influenciada por el Canal del Dique que vierte $397 \mathrm{~m}^{3} \mathrm{~s}^{-1}$ de agua y tiene una carga de sedimentos de $5.9 \times 10^{6}$ ton.año ${ }^{-1}$. Descargas mayores a $800 \mathrm{~m}^{3} \cdot \mathrm{s}^{-1}$ y cargas de sedimento superiores a $31 \times 10^{3}$ ton. día ${ }^{-1}$ se presentan durante noviembre (Restrepo \& Kjerfve 2000, Restrepo et al. 2006), que coinciden con la época lluviosa.

El trabajo de campo se realizó en el arrecife franjeante al norte de Isla Grande en el cual la cobertura de coral vivo se estima en $40 \%$, el sustrato muerto en $25 \%$ y la cobertura algal en 23\% (Zapata 2005). La zona de muestreo se ubicó dentro de la unidad Montastraea spp., entre 3 y 10 metros de profundidad, la cual se caracteriza por exposición al oleaje moderado y donde las especies M. annularis, M. faveolata, Colpophyllia natans y S. siderea son los principales representantes (Díaz et al. 2000). Allí M. annularis, M. faveolata y M. franksi presentan una cobertura media relativa de $42.1 \%$ (Cendales et al. 2002).

Métodos: Este estudio se realizó de septiembre de 2003 a septiembre de 2004. Se escogieron 87 ramets de $M$. annularis (sensu
Weil \& Knowlton 1994) de 24 colonias (cuatro ramets de cada colonia, una perdió el código y no se pudo seguir). Todos los ramets seleccionados al inicio del estudio estaban saludables, con más del $95 \%$ de tejido vivo y un tamaño por encima del reproductivo $\left(>50 \mathrm{~cm}^{2}\right.$ de área, Szmant 1991, Van Veghel \& Kahman 1994). Aunque se trató de escoger un número de ramets representativo para abarcar diferentes tallas, esto no fue posible porque los pocos ramets grandes se encontraban lesionados o estaban en proceso de fisión.

El tamaño de los ramets se determinó mediante filmación del área total de cada una de las columnas en cinco lados (superficie y cuatro lados), hasta donde había evidencia de tejido vivo. Sobre cada ramet se colocó una malla flexible con ojo de malla de $1 \mathrm{~cm}^{2}$. La filmación se realizó cada mes durante un año, excepto en el mes de abril de 2004 (daño de la filmadora), con una cámara de vídeo Sony Handycam. Con el software analizador de imágenes, UTHSCSA Image Tool Versión 3.0, se realizaron las mediciones de los tamaños de los ramets y de las áreas afectadas $\left(\mathrm{cm}^{2}\right)$. Los ramets se clasificaron en 4 clases de talla con escalas de $50 \mathrm{~cm}^{2}$, 1) $0-50 \mathrm{~cm}^{2}$; 2) $51-100 \mathrm{~cm}^{2}$, 3) $101-150 \mathrm{~cm}^{2}$ y 4) $>151 \mathrm{~cm}^{2}$. Aunque los ramets de una misma colonia son clones entre sí, son individuos fisiológicos distintos (Orive 1995) cuya respuesta hacia la reproducción y supervivencia es también diferencial (Hughes $\&$ Tanner 2000). Por lo tanto, en este estudio los ramets fueron considerados como las unidades de muestra.

Las lesiones se definieron como el resultado de disturbio e interacciones naturales de tipo biótico y abiótico, que causan tanto la pérdida de tejido vivo y esqueleto, al igual que la pérdida de color (blanqueamiento) o la aparición de manchas de otro color en la superficie del ramet. Las lesiones identificadas se presentaron por efectos de depredación, interacción directa con organismos reconocidos como potenciales competidores por espacio libre (invasión de tejido coralino por algas, esponjas, bioerodadores), blanqueamiento y enfermedad de la banda amarilla. La identificación de los tipos 
de lesión se basó en las descripciones dadas por Barrios (2000) y para la enfermedad de la banda amarilla por comparación de fotografías de campo con aquellas registradas por Santavy \& Peters (1997). Debido a que durante el año de muestreo las lesiones por banda amarilla solo se presentaron en tres meses (mayo, junio y septiembre) y que las de mayo y junio fueron colonizadas por algas y la lesión que se produjo en septiembre del 2004, no se pudo seguir por ser el último mes de muestreo, en los resultados solo se presenta en casos en que se describe el tipo y la talla de lesión.

Cada lesión de cada uno de los ramets se registró mes a mes y se anotó el tipo de lesión y su tamaño $\left(\mathrm{cm}^{2}\right)$. Con esta información se determinó la presencia-ausencia de lesión, tipo más frecuente de lesión (número), porcentaje de área lesionada por ramet en cada mes, porcentaje de recuperación de lesiones y duración de cada lesión según el tamaño y el tipo. A las que se presentaron en el último mes de muestreo, no se les pudo hacer seguimiento. Las lesiones se cuantificaron según los tamaños encontrados y se clasificaron en siete clases: $0-1 \mathrm{~cm}^{2}, 1.1-5 \mathrm{~cm}^{2}, 5.1-10 \mathrm{~cm}^{2}, 10.1-20 \mathrm{~cm}^{2}$,
$20.1-40 \mathrm{~cm}^{2}, 40.1-60 \mathrm{~cm}^{2}$ y $>60.1 \mathrm{~cm}^{2}$. A pesar de la precisión obtenida por el programa para medir al milímetro más cercano, se estimó un margen de error de $1 \mathrm{~mm}^{2}$. Se elaboró tablas de frecuencia por tipos de lesiones que se recuperaron en el tiempo, que no se recuperaron o que no se pudieron seguir. Las lesiones recuperadas se identificaron como aquellas que presentaron nuevamente pólipos i.e., tejido vivo (Fig. 1) y como no recuperadas, aquellas áreas sin pólipos que permanecieron a lo largo de los meses de duración del estudio y que perdieron el tejido.

Para determinar el efecto de la lesión y la recuperación de tejido sobre la estructura de tallas de la población de ramets monitoreados durante el año, mes a mes se calculó el área de tejido vivo presente. Mediante la construcción de matrices de probabilidades de transición (Caswell 2001) mensuales se determinó cuantos ramets y de qué clases de talla se mantenían en la misma, disminuían a la clase anterior o aumentaban a la siguiente. Con la información de las matrices de transición se construyeron diagramas mes a mes y uno anual para observar los cambios.
A

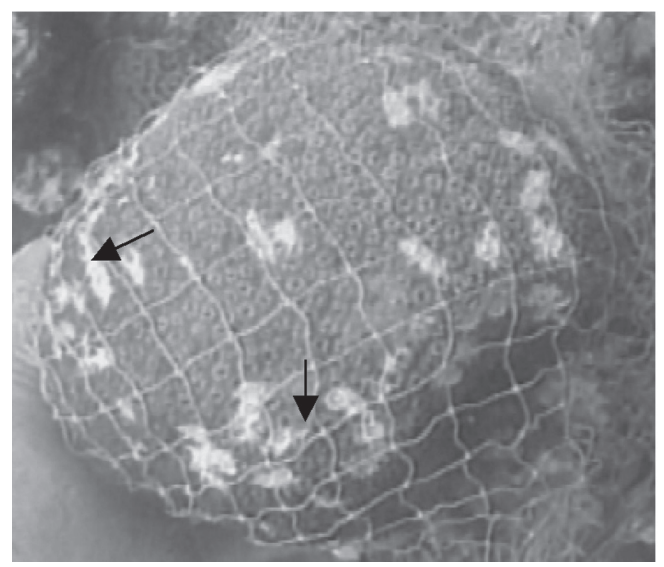

B

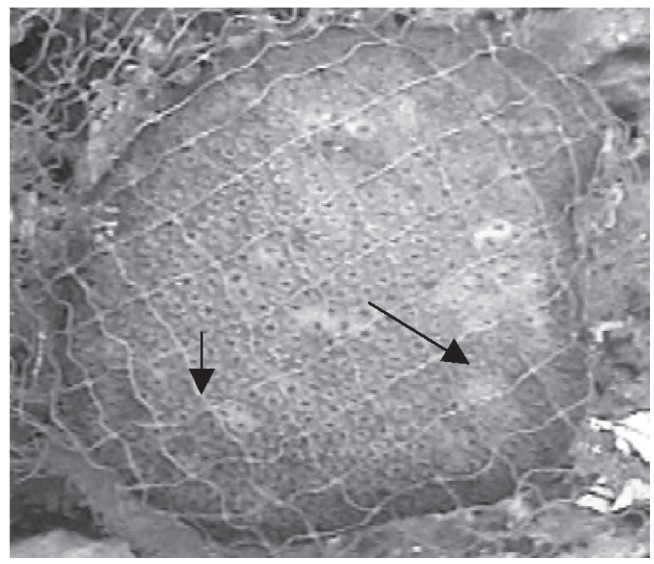

Fig. 1. Lesiones y su recuperación. (a) Ramet con evidencia de varias lesiones causadas por depredación en agosto de 2004. (b) El mismo ramet mostrando recuperación de tejido lesionado en septiembre de 2004. Cada cuadro representa $1 \mathrm{~cm}^{2}$.

Fig. 1. Lesions and recovery. (a) Ramet showing evidence of several lesions caused by predation in August 2004. (b) The same ramet showing tissue recovery, September 2004. Each square represents $1 \mathrm{~cm}^{2}$. 
Debido a que el término regeneración comprende la reconstrucción de partes perdidas o dañadas, o la restauración de tejido y esqueleto (Oren et al. 1997), en este trabajo se utilizó el término de recuperación debido a que se incluyó al blanqueamiento y a la banda amarilla (enfermedad) como tipos de lesión ya que el significado de recuperación involucra el hecho de "volver a retomar o readquirir lo que el tejido inicial antes tenía o parecía, después de haber pasado por una situación adversa" (www.biology-online.org/dictionary/ Right). Adicionalmente se consideró que una lesión no recuperada, era mortalidad parcial en un ramet.

Para el análisis de los datos, previamente se determinó si el tipo de lesión y el número de lesiones que se presentaron en las unidades de muestreo (ramets) estaban asociados a la colonia de origen (4 ramets por cada colonia). Usando como variables nominales al tipo de lesión y la colonia, se hizo una tabla cruzada para determinar la relación entre variables, la cual se comprobó mediante la prueba de Chi-cuadrado de Pearson. Para el caso del número de lesiones y la colonia de origen, los cuatro ramets correspondientes a cada colonia fueron tratados como un bloque y mediante Chi-cuadrado de Pearson se determinó la independencia. Los valores esperados fueron calculados de acuerdo a una tabla de contingencia para la prueba del Chicuadrado. Adicionalmente, mediante la prueba de Friedman se determinó si existían diferencias significativas entre el número de lesiones de los cuatro ramets de cada colonia. Dado que se comprobó que no existe relación entre las variables, y con base en la independencia fisiológica de los ramets, estos se usaron como unidades muestrales diferentes. Así, para determinar si existían diferencias, en el número de lesiones totales en cada ramet y las clases de talla de los ramets $(\mathrm{n}=4)$, así como entre la talla de la lesión y los tipos de lesión $(\mathrm{n}=7)$ se usó la prueba no paramétrica de Kruskall-Wallis. Las diferencias entre grupos se establecieron a partir del diagrama de caja. Por otra parte, la relación entre el número de lesiones según el tipo y el mes fue representada por el modelo de regresión lineal sobre los datos originales. Para observar si existe relación entre el tiempo que dura la recuperación (número de meses) y el tipo de lesión, se utilizó la correlacion de Spearman. La relación entre porcentaje de lesiones recuperadas y la talla del ramet se comprobó mediante la prueba de correlación de Pearson y con la prueba Kendall's Tau b se determinó si existía dependencia entre el porcentaje de área de tejido recuperado y el tipo de lesión. Los análisis se corrieron en los programas Stratgraphics 5.0 y SPSS 10.

\section{RESULTADOS}

De los 87 ramets seguidos en el año de muestreo, seis no presentaron lesión durante todo el periodo de estudio y pertenecían a los ramets de tallas más pequeñas (clases 1 y 2: $<100 \mathrm{~cm}^{2}$ de área). En los 81 ramets que presentaron daño sobre el tejido vivo a través del año, se hallaron seis tipos de lesiones naturales: depredación $(\mathrm{P}, \mathrm{n}=591)$, interacción con algas (IA, $n=80)$, blanqueamiento $(B, n=62)$, interacción con bioerodadores (IB, $\mathrm{n}=12$ ), interacción con esponjas (IE, n=9) y banda amarilla (BA, n=4).

No se encontró relación entre el tipo de lesión presente en los ramets y la procedencia de los ramets según la colonia de origen $\left(\mathrm{p}=0.670, \mathrm{X}^{2}\right.$ de Pearson). De 24 colonias, todas presentaron ramets con depredación, 16 con blanqueamiento y 20 con interacción con algas. Igualmente, el número de lesiones en los diferentes ramets fue independiente de la colonia origen ( $p=0.273, X^{2}$ de Pearson), encontrándose diferencias significativas en el número de lesiones de los cuatro ramets de cada colonia (Friedman, $p=0.04$ ). Entre clases de talla de ramet, también se presentaron diferencias en el número de lesiones (Kruskall-Wallis $\mathrm{p}<0.05$ ), siendo más frecuentes en las clases de talla más grandes (Fig. 2). Los ramets de las clases 1 y 2 fueron más parecidos entre sí y presentaron alrededor de cuatro lesiones (rango 1-12 y $1-19$, respectivamente), mientras que los de las clases 3 y 4 presentaron alrededor de 11 lesiones (rango 2-30, 6-68, respectivamente). 


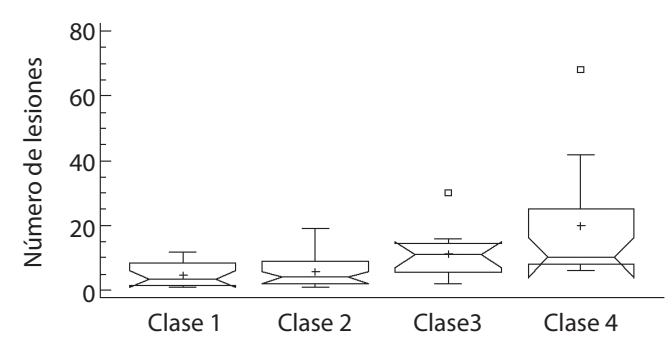

Fig. 2. Diagrama de caja mostrando el número de lesiones promedio por ramet presentes en cuatro clases de talla de ramets. Clase $1\left(0-50 \mathrm{~cm}^{2}\right)$, Clase $2\left(51-100 \mathrm{~cm}^{2}\right)$, Clase 3 $\left(101-150 \mathrm{~cm}^{2}\right)$, Clase $4\left(151-650 \mathrm{~cm}^{2}\right)$.

Fig. 2. Box plot of the average number of lesions per ramet present in four ramet class sizes. Class $1\left(0-50 \mathrm{~cm}^{2}\right)$, Class $2\left(51-100 \mathrm{~cm}^{2}\right)$, Class $3\left(101-150 \mathrm{~cm}^{2}\right)$, Class $4(151-$ $\left.650 \mathrm{~cm}^{2}\right)$.

Lesiones por depredación se presentaron en el $85 \%$ de los ramets y un $88 \%$ de estos exhibieron este tipo de lesión en más de un mes. En el $48 \%$ de los ramets se observaron lesiones por interacción con algas, mientras que aquellas producidas por blanqueamiento afectaron a un $31 \%$. La interacción con bioerodadores se presentó en el $12 \%(\mathrm{n}=10)$, la interacción con esponjas en el $9 \%$ y la banda amarilla en el 4\%. Aquí es necesario recordar que las lesiones por interacción con algas, esponjas y bioerodadores no implican que los organismos hayan causado la pérdida de tejido sino que estos estaban presentes sobre el tejido muerto.

Número de lesiones vs. tipo de lesión: El número de lesiones se encontró dependiente del tipo de lesión $\left(\mathrm{X}^{2}, \mathrm{p}<0.05\right)$. Hubo mayor número de lesiones causadas por depredación (78\%) que por interacción con algas (12\%) y blanqueamiento $(8 \%)$ y estas se presentaron en casi todos los meses. Aquellas producidas por interacción con bioerodadores y con esponjas, en general, solo se presentaron en los primeros meses de muestreo (septiembre a diciembre de 2003) y se mantuvieron o crecieron a lo largo del tiempo. Se encontró una relación estadísticamente significativa $(\mathrm{p}<0.01)$ entre el número de lesiones según el tipo y el mes, representado por el modelo de regresión lineal:

$$
\begin{aligned}
& \text { Número total de lesiones }= \\
& -8.95+8.22 * \text { tipo de lesión }-0.15 * \text { mes }\left(r^{2}=0.38\right) \text {. }
\end{aligned}
$$

Este modelo determinó que la depredación (lesión 6) presenta mayor número de lesiones que la interacción con algas (lesión 3) o que el blanqueamiento (lesión 1). Adicionalmente, se encontró una relación significativa entre el número de lesiones dadas por el blanqueamiento $\left(p=0.08 ; r^{2}=0.43\right.$, Fig. $\left.3 \mathrm{~A}\right)$, la interacción con algas $\left(p=0.01 ; r^{2}=0.74\right.$, Fig. $\left.3 B\right)$, la interacción con esponjas $\left(\mathrm{p}<0.01 ; \mathrm{r}^{2}=0.90\right.$, Fig. $3 \mathrm{C})$ y el mes de ocurrencia. Para lesiones dadas por depredación $\left(\mathrm{p}=0.49 ; \mathrm{r}^{2}=0.15\right.$, Fig. 3D), esta relación no fue significativa. No obstante se encuentra una tendencia a que en los meses cálidos (entre julio y noviembre) se presente un mayor número de lesiones.

Número de lesiones en cada clase de talla de lesión: En general se observó que hay un mayor número de lesiones en tallas pequeñas $\left(<5 \mathrm{~cm}^{2}\right)$ y que el tamaño de las lesiones depende del tipo de lesiones. La mayor cantidad de lesiones por depredación estuvo en el rango del tamaño $0-1 \mathrm{~cm}^{2}$, aquellas producidas por el blanqueamiento, la interacción con algas y la banda amarilla estuvieron en el rango de $1-5 \mathrm{~cm}^{2}$, mientras que la interacción con esponjas presentó una mayor cantidad de lesiones en el rango de $5-10 \mathrm{~cm}^{2}$. Tan solo el blanqueamiento presentó lesiones grandes, por encima de $60 \mathrm{~cm}^{2}$. No obstante, no se encontraron diferencias estadísticas significativas entre las tallas de las lesiones y el tipo de lesion (Kruskall-Wallis $\mathrm{p}>0.05$ ).

Porcentaje de área lesionada en los ramets: $\mathrm{El} 78 \%$ de los ramets mostraron menos de un $10 \%$ del área de tejido afectada por lesiones (Cuadro 1), mientras que un 19\% exhibieron lesiones que afectaron entre el 10 y el $50 \%$ de los tejidos (se destaca el mes de 
Meses

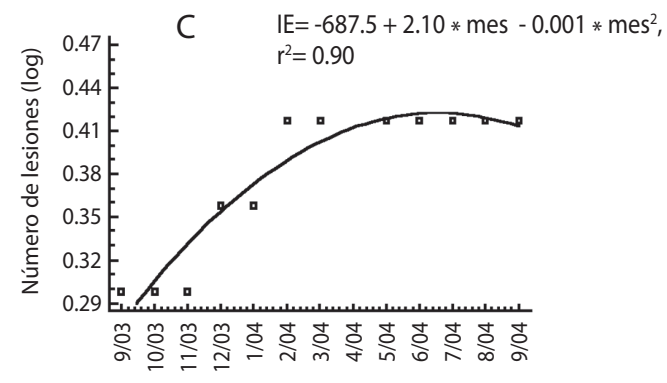

Meses

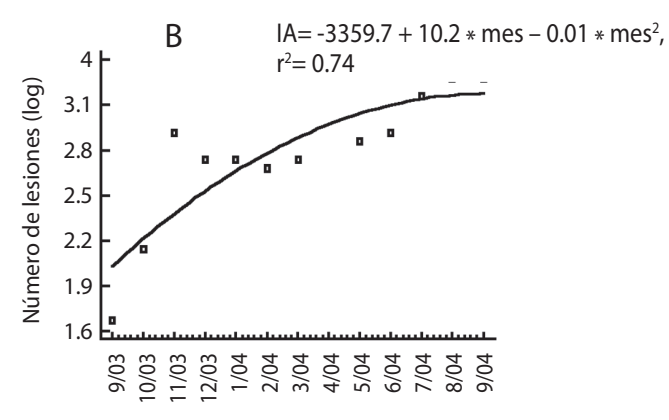

Meses

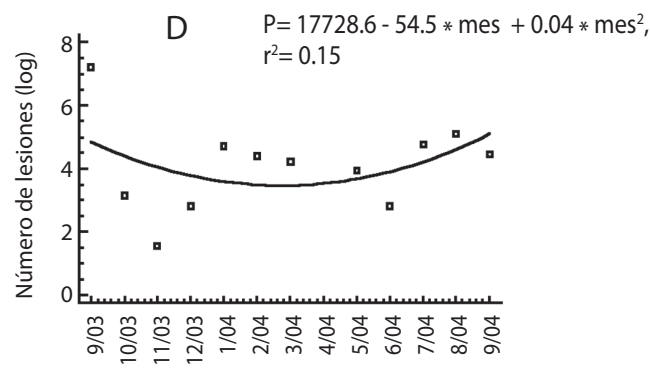

Meses

Fig. 3. Relación entre el número de lesiones totales (porcentaje relativo) según el tipo y el mes de ocurrencia. A) blanqueamiento, B) interacción con algas, C) interacción con esponjas, D) depredación.

Fig. 3. Relationship between number of total lesions (relative percentage) by type and month of occurrence. A) bleaching, B) interaction with algae, C) interaction with sponges, D) predation.

\section{CUADRO 1}

Porcentaje relativo mensual de ramets afectados por lesiones de acuerdo a tres clases porcentuales de áreas de afectación

TABLE 1

Monthly relative percentage of ramets affected by lesions classified in three degrees (\%) of affectation*

\begin{tabular}{cccccccccccccc} 
& \multicolumn{10}{c}{ \% AREA } \\
RAMET & Sep. & Oct. & Nov. & Dic. & Ene. & Feb. & Mar. & May. & Jun. & Jul. & Ago. & Sep. & Total \\
$0-10$ & 95.7 & 71.4 & 80.9 & 76.6 & 77.1 & 81.6 & 77.1 & 77.3 & 77.3 & 70.5 & 76.1 & 74.5 & 78.1 \\
$11-50$ & 4.3 & 19.1 & 17.0 & 17.0 & 18.8 & 14.3 & 20.8 & 22.7 & 22.7 & 27.3 & 21.7 & 23.4 & 19 \\
$51-100$ & & 9.5 & 2.1 & 6.4 & 4.2 & 4.1 & 2.2 & & & 2.3 & 2.2 & 2.1 & 2.9
\end{tabular}

*Los resultados en las filas se refieren al porcentaje de ramets afectados en cada clase de área lesionada.

*The results in the rows show the percentage of ramets affected for each class of injured area. 
julio). Tan solo el 3\% de los ramets presentaron lesiones que afectaron más de la mitad de su área de tejido vivo, siendo particularmente alto en octubre y diciembre. El porcentaje de área relativa de tejido vivo afectado en cada mes por los diferentes tipos de lesión, en relación con el área total de cobertura de tejido de todos los ramets (Cuadro 2), fue baja para aquellas de depredación $(0.8+/-0.4 \mathrm{STD}$, máximo $1.44 \%$ en septiembre de 2004). En la interacción con algas y blanqueamiento el porcentaje de área afectada fue mayor (1.6 +/-0.7 STD, máximo $2.46 \%$ en septiembre de $2004 ; 1.3 \%+/-1.6$ STD, máximo 4.17 en diciembre de 2003, respectivamente).

Recuperación de lesiones según el tipo y tamaño de lesión: El 83\% de las lesiones se recuperaron mientras que el $11 \%$ no se recuperó (Cuadro 3). Las lesiones causadas por depredación mostraron el mayor porcentaje relativo de recuperación (71.6\%), mientras que de las lesiones que no se recuperaron, el mayor porcentaje relativo fue de interacción con algas $(6.7 \%)$. Se determinó que existe dependencia entre el tipo de lesión y el porcentaje de recuperación ( $p=0.97$, Kendall's Tau b), ya que las lesiones producidas por blanqueamiento y por depredación presentaron mayor tendencia a recuperarse que aquellas producidas por la interacción, especialmente con algas. En general, las lesiones producidas por blanqueamiento se recuperaron en $100 \%$ independientemente de su tamaño (Cuadro 4), mientras que las producidas por depredación se recuperaron cuando la lesión era pequeña $\left(<1 \mathrm{~cm}^{2}\right)$. Las lesiones causadas por interacción con algas presentaron una menor recuperación, mientras que aquellas causadas por bioerodadores y esponjas solo se recuperaron cuando las lesiones eran de menor tamaño $\left(1 \mathrm{~cm}^{2}\right)$. Adicionalmente un $2.51 \%$ de las lesiones causadas por estas interacciones no solo no se recuperaron, sino que cambiaron de tipo de lesión. Por ejemplo, 13 lesiones que iniciaron como depredación, 3 como blanqueamiento y 3 como banda amarilla fueron

CUADRO 2

Porcentaje relativo del área lesionada por tipo de lesión*, respecto al área total de todos los ramets entre septiembre de 2003 y septiembre de 2004

TABLE 2

Relative percentage of injured area between September 2003 and September 2004, according to type of lesion*, in relation to total area of all ramets

$\begin{array}{cccccccc}\text { Mes inicio } & \text { B } & \text { IA } & \text { IB } & \text { IE } & \text { P } & \text { Total } \\ \text { Sep-03 } & 0.09 & 0.38 & 0.01 & 0.16 & 0.50 & 1.14 \\ \text { Oct-03 } & 4.10 & 0.64 & 0.02 & 0.20 & 0.26 & 5.21 \\ \text { Nov-03 } & 3.13 & 0.92 & 0.02 & 0.21 & 0.09 & 4.37 \\ \text { Dic-03 } & 4.17 & 1.04 & 0.02 & 0.32 & 0.31 & 5.87 \\ \text { Ene-04 } & 1.55 & 1.53 & 0.02 & 0.72 & 1.06 & 4.88 \\ \text { Feb-04 } & 1.30 & 1.79 & 0.02 & 0.91 & 1.26 & 5.28 \\ \text { Mar-04 } & 0.61 & 1.99 & 0.02 & 1.29 & 0.75 & 4.66 \\ \text { May-04 } & 0.00 & 2.02 & 0.03 & 1.30 & 0.57 & 4.02 \\ \text { Jun-04 } & 0.02 & 2.15 & 0.03 & 1.30 & 0.60 & 4.10 \\ \text { Jul-04 } & 0.09 & 2.34 & 0.03 & 1.30 & 0.86 & 4.62 \\ \text { Ago-04 } & 0.17 & 2.29 & 0.03 & 1.30 & 1.19 & 4.98 \\ \text { Sep-04 } & 0.26 & 2.46 & 0.04 & 1.30 & 1.44 & 5.51\end{array}$

* (B) blanqueamiento, (IA) interacción con algas, (IB) interacción con bioerodadores, (IE) interacción con esponjas, y (P) depredación. Área total de todos los ramets $=9494.6 \mathrm{~cm}^{2}$.

* (B) Bleaching, (IA) interaction with algae, (IB) interaction with burrowers, (IE) interaction with sponges, and, (P) predation. Total area of all ramets $=9494.6 \mathrm{~cm}^{2}$. 
CUADRO 3

Número (\#) y porcentaje relativo (\%) de lesiones que se recuperaron(R) o no (NR)

TABLE 3

Number (\#) and relative percentage (\%) of recovered ( $R$ ) and not recovered lesions (NR)

\begin{tabular}{ccccc} 
Tipo lesión* & \multicolumn{2}{c}{ Recuperadas (R) } & \multicolumn{2}{c}{ No recuperadas (NR) } \\
B & $\#$ & $\%$ & $\#$ & \% \\
IA & 55 & 7.3 & 0 & 0 \\
IB & 25 & 3.3 & 51 & 1.3 \\
IE & 2 & 0.3 & 10 & 0.9 \\
P & 2 & 0.3 & 7 & 2.2 \\
Total & 543 & 71.6 & 17 & 11.1
\end{tabular}

* (B) Blanqueamiento, (IA) interacción con algas, (IB) interacción con bioerodadores, (IE) interacción con esponjas, y (P) depredación. El restante $6.07 \%$ corresponde a lesiones que no se pudieron seguir $(3.56 \%)$ o que cambiaron de un tipo de lesión a otro $(2.51 \%)$.

* (B) Bleaching, (IA) interaction with algae, (IB) interaction with burrowers, (IE) interaction with sponges and, (P) predation. The additional $6.07 \%$ corresponds to lesions that were not followed $(3.56 \%)$ or that changed from one type of lesion to another $(2.51 \%)$.

\section{CUADRO 4}

Porcentaje del número de lesiones recuperadas $(R, n=627)$ y no recuperadas $(N R, n=85)$ en cinco tipos de lesiones (porcentaje relativo)*, según la talla inicial de lesión $\left(\mathrm{cm}^{2}\right)$

TABLE 4

Percentage of number of lesions that recovered $(R, n=627)$ or did not $(N R, n=85)$ grouped by five types of lesions (relative percentage) * regarding the initial size of the lesion $\left(\mathrm{cm}^{2}\right)$

\begin{tabular}{|c|c|c|c|c|c|c|c|c|c|c|c|c|}
\hline \multicolumn{2}{|c|}{ Tamaño lesión } & \multicolumn{6}{|c|}{ Recuperadas (R) } & \multicolumn{5}{|c|}{ No recuperadas (NR) } \\
\hline Clase & Rango & B & IA & IB & IE & $P$ & Total & IA & IB & IE & $\mathrm{P}$ & Total \\
\hline 1 & $0-1$ & 0.92 & 2.90 & 0.26 & 0.26 & 63.46 & 67.81 & 3.17 & 1.32 & & 0.53 & 5.01 \\
\hline 2 & $1-5$ & 2.90 & 0.40 & & & 7.92 & 11.21 & 2.37 & & 0.66 & 1.32 & 4.35 \\
\hline 3 & $5-10$ & 1.45 & & & & 0.26 & 1.72 & 0.66 & & 1.13 & & 0.79 \\
\hline 4 & $10-20$ & 0.92 & & & & & 0.92 & 0.40 & & 1.13 & 0.13 & 0.66 \\
\hline 5 & $20-40$ & 0.66 & & & & & 0.66 & 0.13 & & & 0.26 & 0.40 \\
\hline 6 & $40-80$ & 0.40 & & & & & 0.40 & & & & & 0.00 \\
\hline & & 7.26 & 3.30 & 0.26 & 0.26 & 71.64 & 82.72 & 6.73 & 1.32 & 0.92 & 2.24 & 11.21 \\
\hline
\end{tabular}

* (B) Blanqueamiento, (IA) interacción con algas, (IB) interacción con bioerodadores, (IE) interacción con esponjas, (P) depredación. El restante $6.07 \%$ corresponde a lesiones que no se pudieron seguir $(3.56 \%)$ o que cambiaron de un tipo a otro $(2.51 \%)$.

* (B) Bleaching, (IA) interaction with algae, (IB) interaction with burrowers, (IE) interaction with sponges, and, (P) predation. The additional $6.07 \%$ corresponds to lesions that were not followed (3.56\%) or that changed from one type of lesion to another $(2.51 \%)$. 
colonizadas posteriormente por algas y las lesiones no se recuperaron.

Se observó una tendencia a que a menor tamaño de lesión, mayor recuperación. En el Cuadro 5 se puede ver que la mayoría de las lesiones en clases de talla pequeñas, independientemente del tipo de lesión, se recuperaron. En tallas mayores, si bien el porcentaje de frecuencia de lesiones es bajo, se observó una tendencia a que la lesión permaneciera.

Duración de las lesiones: La duración de las lesiones en general fue corta ya que el $44 \%$ de las lesiones se recuperaron en menos de un mes y cerca del $70 \%$ permanecieron menos de tres meses. Se encontró correlación entre el tiempo que dura la recuperación (número de meses) y el tipo de lesión (Spearman's rho, $\mathrm{p}<0.01$ ). Más del $50 \%$ de las lesiones causadas por depredación y blanqueamiento se recuperaron en el primer mes, mientras que las lesiones causadas por interacción con algas, bioerodadores y esponjas permanecieron en el tiempo. En estas últimas se encontró que la duración es dependiente del tipo de lesión.

Recuperación de lesiones según las clases de talla de los ramets: Del $83 \%$ de ramets que recuperaron las lesiones, se observó que a mayor tamaño del ramet, mayor recuperación, independientemente del tipo de lesión (Cuadro 5). Esta relación, fue significativa $(\mathrm{p}<0.05)$ pero débil $\left(\mathrm{r}^{2}=0.19\right.$, correlación de Pearson).

Dinámica anual en la estructura de tallas de ramets dadas por lesión y recuperación: Aunque la mayoría de los ramets se mantuvieron en la misma categoría de talla de ramet a través del año, entre 17 y 19 ramets disminuyeron sus tallas a una clase menor en cada mes. Incluso, entre los meses de octubre y febrero hubo ramets que disminuyeron entre 5 y 8 tallas. Al comparar la estructura de tallas de ramets entre el inicio y el final de estudio se encontró que 18 ramets disminuyeron sus tallas en al menos una clase siendo las probabilidades de transición mayores en las clases de talla 5 y 6 (0.04 y 0.02, respectivamente, Fig. 4).

\section{DISCUSIÓN}

Los resultados de este estudio muestran que $M$. annularis presenta lesiones naturales como una característica permanente en los ramets y confirma resultados anteriores para

CUADRO 5

Porcentaje relativo del número de lesiones recuperadas según el tipo de lesión* en relación con las clases de talla de ramet $\left(\mathrm{cm}^{2}\right)$

TABLE 5

Relative percentage of number of recovered lesions grouped according to lesion type* in relation to ramet class size $\left(\mathrm{cm}^{2}\right)$

\begin{tabular}{|c|c|c|c|c|c|c|c|}
\hline \multicolumn{2}{|c|}{ Tamaño ramet } & \multicolumn{6}{|c|}{ Lesiones recuperadas } \\
\hline Clase & Rango & $\mathrm{B}$ & IA & IB & IE & $P$ & Total \\
\hline 1 & $1-50$ & 0.5 & 0.1 & & & 7.5 & 8.2 \\
\hline 2 & $51-100$ & 2.4 & 0.5 & 0.1 & & 17.2 & 20.2 \\
\hline 3 & $101-150$ & 2.1 & 0.4 & & 0.3 & 11.7 & 14.5 \\
\hline 4 & $>150$ & 2.2 & 2.2 & 0.1 & & 35.2 & 39.8 \\
\hline \multicolumn{2}{|c|}{ Total } & 7.3 & 3.3 & 0.2 & 0.3 & 71.6 & 82.7 \\
\hline
\end{tabular}

* (B) Blanqueamiento, (IA) interacción algas, (IB) interacción con bioerodadores, (IE) interacción con esponjas, (P) depredación.

* (B) Bleaching, (IA) interaction with algae, (IB) interaction with burrowers, (IE) interaction with sponges, and, (P) predation. 


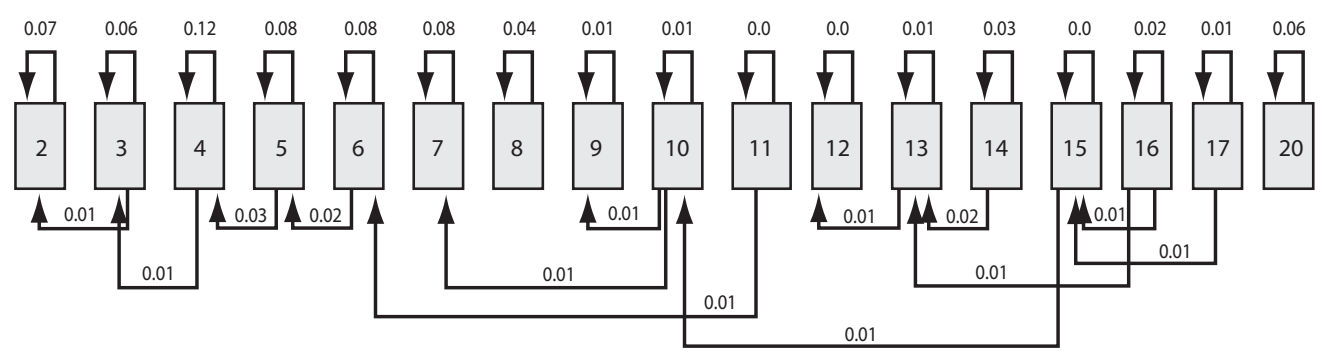

Fig. 4. Diagrama de probabilidades de transición entre clases de talla de ramets de M. annularis durante un año. Las flechas superiores indican mantenimiento en la misma clase y las inferiores, la probabilidad de disminución de talla. No se observó crecimiento a la siguiente clase. Como no hubo ramets en clase de talla 1, 18 y 19, estos no se incluyeron en el diagrama.

Fig. 4. Transition probabilities diagram between M. annularis ramet class sizes in a year. Upper arrows indicate no change in size (same class), and lower arrows, the probability of decreasing size. Growth to the next class was not observed. Since ramets of classes 1, 18 and 19 were not observed, these were not included in the diagram.

esta especie (Meesters et al. 1997, Sánchez et al. 2004; Chasqui et al. 2007). El efecto del tipo de lesiones sobre los ramets se podría caracterizar por la abundancia, el tamaño y la frecuencia con la consecuente respuesta a la recuperación de la lesión y por ende a la mortalidad parcial. Por ejemplo, el efecto de la lesión dado por la depredación en los ramets es significativo, ya que aunque de magnitud pequeña (e.g. en un mes como septiembre del 2004 afectó cerca del 1.4\% del área total de los ramets y en todo el año cerca del $9 \%$ del área total de los ramets), es frecuente y abundante y de recuperación rápida (<tres meses). Además, en unos pocos casos en los que las lesiones fueron grandes, se presentó mortalidad parcial en los ramets ya que no hubo recuperación pero sí invasión de algas. En lesiones asociadas con la interacción con algas (frecuentes y pequeñas $<5 \mathrm{~cm}^{2}$ ) o esponjas (poco frecuentes y medianas $>5 \mathrm{~cm}^{2}$ ), que afectaron un porcentaje de área de 2.46 y 1.30 del total del área de los ramets, respectivamente, el hecho de que no se recuperara el tejido de coral, representó mortalidad parcial y disminución en la talla de algunos ramets. Al respecto, se ha determinado que la pérdida crónica de tejido causada por depredación, colonización por otros organismos y necrosis en porcentajes tan bajos como $0.4 \%$ en la cobertura de coral en $M$. annularis pueden ser indetectables en monitoreos corrientes y por lo tanto parecer poco representativos. Sin embargo, estos pueden representar hasta el $86 \%$ de la tasa de mortalidad de ramets enteros en periodos cortos de tiempo (Bythell et al. 1993), de tal manera que no solo afectan el crecimiento y estado de salud de los individuos (Neudecker 1979, Miller \& Hay 1998) sino que también disminuyen las tallas de los ramets (o colonias), con lo cual se puede afectar la estructura de tallas de la población.

Adicionalmente, la constante acción depredadora sobre los ramets y la mayor ocurrencia de lesiones causadas por interacciones implican continua asignación energética hacia la reparación de tejido (Meesters et al. 1997). Estudios anteriores muestran que las colonias individuales tienen una capacidad restringida para la regeneración debido a que existe una reserva celular y energética limitada (Meszaros \& Bigger 1999, Mullen et al. 2004) la cual es asignada preferencialmente hacia la reparación i.e., mantenimiento y supervivencia del individuo (Oren et al. 2001). Por otra parte, la recuperación de una lesión también implica un incremento en el riesgo de colonización por algas epilíticas o bioerodadores (Bak \& Steward-Van Es 1980) y esponjas incrustantes (Schonberg \& Wilkinson 2001). Si se tiene en cuenta que la pérdida extensiva de tejido resulta en muerte 
de pólipos dentro de un área dada y que el área de tejido y volumen representa el potencial para alimentación, respiración, reproducción, crecimiento y mantenimiento del individuo, entonces la pérdida de área (pólipos) tiene profundas implicaciones en la supervivencia y reproducción de los ramets (Hall 1997).

A pesar de que las lesiones producidas por blanqueamiento fueron poco frecuentes y de corta duración, afectaban gran porcentaje del área viva de unos pocos ramets. A diferencia de las otras lesiones, en este caso no hubo pérdida de tejido o esqueleto de coral. No obstante, la disminución de zooxantelas implica disminución de energía disponible para la realización de las funciones vitales dado que el metabolismo del individuo es afectado por la pérdida del suplemento del carbono al coral (Hayes \& Bush 1990, Mascarelli \& BunkleyWilliams 1999, Edmunds et al. 2003). Si esta lesión puede llegar a representar el $4.2 \%$ del área total de ramets afectada en un mes dado (e.g. diciembre de 2003) y se presenta en unos pocos ramets que no tendrán nutrientes para el crecimiento o para producir gametos (Edmunds et al. 2003), entonces también se puede sugerir que, a pesar de la recuperación, podría haber efectos en la estructura de la población.

Teniendo en cuenta que con el calentamiento global se predice que las afecciones a los corales causadas por las interacciones con esponjas incrustantes serán más frecuentes (Weil et al. 2002), que la tasa de crecimiento de las esponjas aumentará con el aumento de la temperatura (López-Victoria \& Zea 2005) y, si las lesiones relacionadas con esta interacción no sólo no se recuperan sino que tienden a crecer (este estudio), entonces se puede esperar que la mortalidad parcial de los ramets a consecuencia de esta interacción va a ser cada vez mayor. De ser así, se prevé que con el aumento en la frecuencia en intensidad de los fenómenos de calentamiento de agua (Hoegh-Gulberg 1999) y blanqueamiento masivo, esta población podría presentar una mayor mortalidad de los ramets.

Algunas lesiones que no se recuperaron por depredación y blanqueamiento fueron colonizadas por algas, incrementando el porcentaje de afección anual por este tipo de lesión en un $2 \%$ adicional. Parece ser entonces que el efecto de la interacción con algas es un causante importante de la mortalidad parcial en los ramets de la población de M. annularis en el arrecife de Isla Grande. Para autores, como Cróquer et al. (2002) y Sánchez et al. (2004), en $M$. annularis las lesiones que implican remoción de tejido tienen alta probabilidad de recuperación mientras que las que incluyen destrucción de tejido esquelético y pérdida de tejido pueden tomar más tiempo o no recuperarse si son sobrecrecidas por algas. Aunque en el presente estudio no se observó el agente causante de las lesiones por depredación, se observó que cuando la lesión parecía haber sido causada por un pez mariposa (pequeña y $\sin$ afectar el esqueleto) había una mayor recuperación.

La duración de las lesiones fue corta ya que el $44 \%$ de ellas se recuperaron en menos de un mes mientras que para el tercer mes el $70 \%$ lo cual reafirma los resultados obtenidos para M. annularis en otros estudios (Meesters \& Bak 1993, Meesters et al. 1992, 1994, 1997, Sánchez et al. 2004). Por otra parte, la tasa de recuperación disminuyó con el tiempo y mostró una tendencia al cese de recuperación de área de tejido lesionado a partir del quinto mes, coincidiendo con otros estudios (e.g., Kramarsky-Winter \& Loya 2000). Sin embargo, la mayoría de las investigaciones anteriores siguieron lesiones simuladas (artificiales) por depredación y no contemplaron las ocasionadas por interacciones con especies invasoras como algas y esponjas. Estas lesiones, en el presente estudio, mostraron una clara tendencia hacia la no-recuperación y al aumento en la extensión de la lesión, causando mayor mortalidad parcial en los ramets. Aunque no se encontró relación entre la talla del ramet y la recuperación de lesiones, sí se observó una tendencia de mayor recuperación de lesiones cuando las tallas de los ramets eran mayores, lo cual ha sido atribuido a que los corales grandes tienen mayor energía y tejido de reserva y pueden experimentar más rápida reparación que los 
corales pequeños (Kramarsky-Winter \& Loya 2000, Oren et al. 2001).

Si bien los ramets, en general, no presentaron más del $10 \%$ de su tejido vivo afectado por algún tipo de lesión en cada mes, se pudo observar que de un $95 \%$ de ramets con afección en el primer mes de muestreo, un $25 \%$ de los ramets tuvo un mayor porcentaje de tejido afectado un año más tarde. Es decir que en un periodo de un año hubo mortalidad parcial en los ramets lo cual se puede explicar por la norecuperación de las lesiones relacionadas con interacción con algas y esponjas. No obstante que la frecuencia de lesiones causadas por este tipo de interacciones sea baja, la talla de las lesiones y la tendencia a la no-recuperación y al crecimiento de las mismas podría estar explicando los patrones de mortalidad parcial y de deterioro que presenta la especie en Colombia (Zea et al. 1998, Garzón-Ferreira et al. 2005) y el Caribe (Klomp 2000, Ginsburg et al. 2001). De hecho, Ginsburg et al. (2001) reportan que el $60 \%$ de los corales de "clusters" de $M$. annularis presentaron entre el 40 y $70 \%$ de la superficie de tejido muerto, lo cual afecta la estructura de la población ya que probablemente esto implica muerte total de ramets pequeños y alta mortalidad parcial de ramets más grandes. La población de M. annularis en el arrecife de Isla Grande presentó más del 50\% de cobertura de tejido muerto (Alvarado et al. en prep.). Si en un año aumenta la mortalidad parcial en $25 \%$ de los ramets sanos, principalmente en los ramets de mayor talla (reproductivos), es posible pensar que la población presenta signos de declinamiento dado por el disturbio crónico de lesiones como depredación, interacción con algas y con esponjas.

Una especie como $M$. annularis con tasas de crecimiento lento (Charry et al. 2004) y presente en localidades con disturbios crónicos por depredación (este estudio) y sedimentación (Restrepo et al. 2006), así como con eventos de blanqueamiento cada vez más intensos y frecuentes (Gardner et al. 2003, Edmunds \& Elahi 2007) probablemente no tenga tiempo para recuperarse entre eventos tan seguidos. De ser así es probable que la baja cobertura coralina dada por alta mortalidad parcial y blanqueamiento continuo, sean una de las razones para la pérdida de cobertura coralina en los últimos años (Williams \& Bunkley-Williams 1990). Para el caso presente, el blanqueamiento no parece estar causando mortalidad parcial, pero la interacción con algas especialmente, podría ser uno de los causantes de la alta mortalidad.

En general se concluye que las lesiones que impliquen interacciones con algas, esponjas y bioerodadores que por lo tanto determinen una interacción de competencia entre el coral y los otros grupos, causan lesiones que en general tienden a permanecer en el tiempo y que por lo tanto afectan la estructura de talla de los ramets, por disminución de las tallas, pérdida de tejido vivo y por lo tanto, el número de pólipos que puedan aportar a la supervivencia y la reproducción.

\section{AGRADECIMIENTOS}

Deseamos agradecer a D. L. Duque por su permanente colaboración en campo. A M. Rozo por la medición de las lesiones a través de los meses. M. A. Rodríguez y C. Barreto por su asesoría en la parte estadística. Al personal del Parque Corales del Rosario por las facilidades logísticas y su interés en que el proyecto se pudiera realizar. A F. Zapata, V. Pizarro, J. Cantera y J. Torres, por la revisión y evaluación del manuscrito que forma parte de la tesis doctoral del primer autor, así como a los evaluadores anónimos quienes con sus correcciones y sugerencias mejoraron este documento. Permiso de Investigación DTCA023- UAESPNN.

\section{RESUMEN}

Las poblaciones de Montastraea annularis muestran síntomas de declinamiento y sin embargo, pocos estudios se han realizado sobre tipos, frecuencias e intensidad de lesiones naturales in situ. Durante un año, septiembre 2003 a septiembre 2004, se hizo el seguimiento anual de lesiones naturales que aparecieron en ramets sanos de una población en un arrecife degradado dentro de un Área Marina Protegida, para inferir su efecto en la estructura de talla de la población. El 94\% de los ramets presentaron lesiones causadas por blanqueamiento, depredación e interacciones 
con algas, esponjas y bioerodadores. La depredación causó el $47 \%$ y la interacción con algas el 36\%. El $85 \%$ de las lesiones eran de tallas pequeñas $\left(<5 \mathrm{~cm}^{2}\right)$ aunque algunas fueron mayores a $60 \mathrm{~cm}^{2}(0.3 \%)$. La recuperación de las lesiones fue alta (83\%), sin embargo $11.1 \%$ no se recuperaron y por el contrario aumentaron. En el $2.5 \%$ de las lesiones hubo invasión de algas. Las lesiones causadas por blanqueamiento afectaron mayor área de los ramets $(>60 \%$ de su tejido vivo) en los meses de septiembre a noviembre, pero la recuperación fue del $100 \%$ a los tres meses. Por el contrario, las lesiones con algas (36\%), mostraron menor recuperación $(6.7 \%)$ y una tendencia a aumentar el área de lesión con el tiempo. En general, el porcentaje de área de tejido afectada por lesiones en un ramet durante cualquier mes fue menor a $10 \%$. Sin embargo, de mayo a septiembre el porcentaje de tejido afectado fue mayor (10-50\%) debido al incremento en la frecuencia y abundancia de depredación, blanqueamiento e interacción con algas, y a la menor tasa de recuperación. Al final de un año, las lesiones que no se recuperaron causaron mortalidad parcial en el $25 \%$ de los ramets. El efecto de las lesiones y la recuperación sobre el tamaño de los ramets evidenció disminución de talla a lo largo del año, y al final de este, significó que el $21 \%$ pasara a una clase de talla menor.

Palabras clave: lesiones naturales, recuperación, blanqueamiento, depredación, Montastraea annularis, mortalidad parcial, estructura de clases de talla.

\section{REFERENCIAS}

Alvarado, E.M, G. Pinilla, T. León, E. Sarmiento, F. Flechas, G. Alvis, G. Vargas, F. Arias, R. Steer \& A. Ramos. 1992. Plan de Manejo para el Parque Nacional Natural Corales del Rosario (Cartagena, Colombia). Bol. Ecotrópica: Ecosistemas Trop. 1: 33-37.

Bak, R.P.M. \& Y. Steward-Van Es. 1980. Regeneration of superficial damage in the scleractinian corals Agaricia agaricites, F. purpurea and Porites astreoides. Bull. Mar. Sci. 30: 883-887.

Barrios, L. 2000. Evaluación de las principales condiciones de deterioro de los corales pétreos en el Caribe colombiano. Tesis de Maestría. Universidad Nacional de Colombia. Colombia.

Bythell, J.C., E.H. Gladfelter \& M. Bythell. 1993. Chronic and catastrophic natural mortality of 3 common Caribbean reef corals. Coral Reefs 12: 143-152.

Bythell, J., Z. Hillis-Starr \& C. Rogers. 2000. Local variability but landscape stability in coral reef communities following repeated hurricane impacts. Mar. Ecol. Prog. Ser. 204: 93-100.
Caswell, H. 2001. Matrix population models. Sinauer. Massachussetts, EEUU.

Cendales, M.H., S. Zea \& J.M. Díaz. 2002. Geomorfología y unidades ecológicas del complejo de arrecifes de las Islas del Rosario e Isla Barú (Mar Caribe, Colombia). Rev. Acad. Col. Cien. 26: 497-510.

Charry, H., E.M. Alvarado \& J. Sánchez. 2004. Annual skeletal extension of two reef-building corals from the Colombian Caribbean Sea. Bol. Invest. Mar. Cost. (INVEMAR) 33: 207-220.

Chasqui, L.H, E.M. Alvarado, A. Acero \& F. Zapata. 2007. Efecto de la herbivoría y coralivoría por peces en la supervivencia de corales transplantados en el Caribe Colombiano. Rev. Biol. Trop. 55: 825-837.

Connell, J.H., T.P. Hughes \& C.C. Wallace. 1997. A 30-year study of coral abundance, recruitment, and disturbance at several scales in space and time. Ecol. Monogr. 67: 461-488.

Cróquer, A., E. Villamizar \& N. Noriega. 2002. Environmental factors affecting regeneration of the reef-building coral Montastraea annularis (Faviidae) at Los Roques National Park, Venezuela. Rev. Biol. Trop. 50: 1055-1065.

Diaz, J.M., L.M. Barrios, M.H. Cendales, J. GarzónFerreira, J. Geister, M. López-Victoria, G.H. Ospina, F. Parra-Velandia, J. Pinzon, B. Vargas-Angel, F.A. Zapata \& S. Zea. 2000. Areas Coralinas de Colombia. INVEMAR. Serie de Publicaciones Especiales, No 5, Santa Marta, Colombia.

Edmunds, P. 2002. Long-term dynamics of coral reefs in St. John, US Virgin Islands. Coral Reefs 21: 357-367.

Edmunds, P., R. Gates \& D. Gleason. 2003. The tissue composition of Montastraea franksi during a natural bleaching event in the Florida Keys. Coral Reefs 22: 54-62.

Edmunds, P. \& R. Elahi. 2007. The demographics of a 15- year decline in cover of the Caribbean reef coral Montastraea annularis. Ecol. Monogr. 77: 3-18.

Fabricius, K. 1995. Slow population turnover in the soft coral genera Sinularia and Sarcophyton on mid- and outer-shelf on the Great Barrier Reef. Mar. Ecol. Prog. Ser. 126: 145-152.

Fong, P. \& P.W. Glynn. 1998. A dynamic size-structured population model: does disturbance control size- structure of a population of the massive coral Gardineroseris planulata in the Eastern Pacific? Mar. Biol. 130: 663-674. 
Fong, P. \& W. Glynn. 2000. A regional model to predict coral population dynamics in response to El NiñoSouthern Oscillation. Ecol. Appl. 10: 842-854.

Gardner, T.A., I.M. Côté, J.A. GilL, A. Grant \& A.R. Watkinson. 2003. Long-term region-wide declines in Caribbean corals. Science 301: 958- 960.

Garzón-Ferreira, J., D. Gil-Agudelo, L. Barrios \& S. Zea. 2001. Stony coral diseases observed in southwestern Caribbean Reefs. Hydrobiologia 469: 65-69.

Garzón-Ferreira, J., S. Zea \& J.M. Díaz. 2005. Incidence of partial mortality and other health indicators in hardcoral communities of four southwestern Caribbean atolls. Bull. Mar. Sci. 76: 105-122.

Garren, M., S. Walsh, A. Caccone \& N. Knowlton. 2006. Patterns of association between Symbiodinium and members of the Montastraea annularis complex on spatial scales ranging from within colonies to between geographic regions. Coral Reefs 25: 503-512.

Ginsburg, R., E. Gischeler \& W. Kiene. 2001. Partial mortality of massive reef-building corals. An index of patch reef conditions, Florida Reef tract. Bull. Mar. Sci. 69: 1149-1173.

Guzmán, H., K. Burns \& J. Jackson. 1994. Injury, regeneration and growth of Caribbean reef corals after a major oil spill in Panama. Mar. Ecol. Prog. Ser. 105: 231-241.

Hall, V. 1997. Interspecific differences in the regeneration of artificial injuries on scleractinian corals. J. Exp. Mar. Biol. Ecol. 212: 9-23.

Hayes, R. \& P. Bush. 1990. Microscopic observations of recovery in the reef-building scleractinian coral Montastraea annularis, after bleaching on a Cayman reef. Coral Reefs 8: 203-209.

Hoegh-Guldberg, O. 1999. Climate change, coral bleaching and the future of the world's coral reefs. Mar. Fresh. Res. 50: 839-866.

Hughes, T.P. \& J.E. Tanner. 2000. Recruitment failure, life histories and long-term decline of Caribbean corals. Ecology 81: 2250-2263.

Klomp, K. 2000. AGRRA assesses Jamaican reef. Reef Encounters 28: 15-16.

Kramarsky-Winter, E. \& Y. Loya. 2000. Tissue regeneration in the coral Fungia granulosa: the effect of extrinsic and intrinsic factors. Mar. Biol. 137: 867-873.

Lewis, J.B. 1997. Abundance, distribution and partial mortality of the massive coral Siderastrea siderea on degrading coral reefs at Barbados, West Indies. Mar. Pollut. Bull. 8: 622-627.

Lirman, D. 2000. Lesion regeneration in the branching coral Acropora palmata: effects of colonization, colony size, lesion size, and lesion shape. Mar. Ecol. Prog. Ser. 197: 209-215.

López-Victoria, M. \& S. Zea. 2005. Current trends of space occupation by encrusting excavating sponges on Colombian coral reefs. Mar. Ecol. 26: 33-41.

Mascarelli, P. \& L. Bunkley-Williams. 1999. Damaged, unbleached and artificially bleached star coral, Montastraea annularis. Bull. Mar. Sci. 65: 577-586.

McCook, L., J. Jompa \& G. Díaz-Pulido. 2001. Competition between corals and algae on coral reefs: a review of evidence and mechanisms. Coral Reefs 19: 400-417.

Meesters, E.H., A. Bos \& G. Gast. 1992. Effects of sedimentation and lesion position on coral tissue regeneration. Proc. $7^{\text {th }}$ Int. Coral Reef Symp. 2: 671-678.

Meesters, E.H. \& R.P.M. Bak. 1993. Effect of coral bleaching on tissue regeneration and colony survival. Mar. Ecol. Prog. Ser. 96: 189-198.

Meesters, E.H., M. Noordeloos \& R.P.M. Bak. 1994. Damage and regeneration: links to growth in the reefbuilding coral Montastraea annularis. Mar. Ecol. Prog. Ser. 112: 119-128.

Meesters, E.H., W. Pauchli \& R.P.M. Bak. 1997. Predicting regeneration of physical damage on a reef-building coral by regeneration capacity and lesion shape. Mar. Ecol. Prog. Ser. 146: 91-99.

Meszaros, A. \& C. Bigger. 1999. Qualitative and quantitative study of wound healing processes in the coelenterate, Plexaurella fusifera: spatial, temporal, and environmental (light attenuation) influences. J. Invertebr. Pathol. 73: 321-331.

Miller, M. \& M. Hay. 1998. Effects of fish predation and seaweed competition on the survival and growth of corals. Oecologia 113: 231-238.

Miller, J., C. Rogers \& R. Waara. 2003. Monitoring the coral disease, plague type II, on coral reefs in St. John, U.S. Virgin Islands. Rev. Biol.Trop. 51: 47-55.

Mullen, K., E. Peters \& C. Harvell. 2004. Coral resistance to disease. p. 377-399. In E. Rosenberg (eds.). Coral health and disease. Springer-Verlag Press. USA.

Mumby, P.J., N.L. Foster \& E.A. Glynn. 2005. Patch dynamics of coral reef macroalgae under chronic and acute disturbance. Coral Reefs 24: 681-692. 
Mumby, P., A. Hastings, \&. H. Edwards. 2007. Thresholds and the resilience of Caribbean coral reefs. Nature 450: 98-101.

Neudecker, S. 1979. Effects of grazing and browsing fishes on the zonation of corals in Guam. Ecology 60: 666-672.

Oren, U., Y. Benayahu \& Y. Loya. 1997. Colony integration during regeneration in the stony coral Favia favus. Ecology 82: 802-813.

Oren, U., Y. Benayahu, H. Lubinesvky \& Y. Loya. 2001. Colony integration during regeneration in the stony coral Favia favus. Ecology 82: 802-813.

Orive, M.E. 1995. Senescence in organisms with clonal reproduction and complex life histories. Am. Nat. 145: $90-108$

Pandolfi, J., C. Lovelock \& A. Budd. 2002. Character release following extinction in a Caribbean reef coral species complex. Evolution 56: 479-501.

Peters, E., N. Gassman, J. Firman, R. Richmond \& E. Power. 1997. Ecotoxicology of tropical marine ecosystems. Environ. Toxicol. Chem. 16: 12-40.

Pujos, M., J. Pagliardini, R. Steer, G. Vernette \& O. Weber.1984. Influence du contre-courant côtier Nord Colombien sur la circulation des eaux du plateau continental: son action sur la dispersion des rejets en suspension du Magdalena. Bull. Inst. Geol. du Bassin d'Aquitaine. 35: 77-85.

Restrepo, J. \& B. Kjerfve. 2000. Magdalena River: interannual variability (1975-1995) and revised water discharge and sediment load estimates. J. Hydrol. 235: $137-149$.

Restrepo, J., P. Zapata, J. Díaz, J. Garzón-Ferreira \& C. García. 2006. Fluvial fluxes into the Caribbean Sea and their impact on coastal ecosystems: the Magdalena River, Colombia. Global and Planet Change 50: 33-49.

Rogers, C., T. Suchanek \& F. Pecora. 1982. Effects of hurricanes David and Frederic (1979) on shallow Acropora palmata reef communities: St. Croix, U. S. Virgin Islands. Bull. Mar. Sci. 32: 532-548.

Rowan, R., N. Knowlton, A. Baker \& J. Jara. 1997. Landscape ecology of algal symbionts creates variation in episodes of coral bleaching. Nature. 388: 265-269.

Ruesink, J. 1997. Coral injury and recovery: matrix model link process to pattern. J. Exp. Mar. Biol. Ecol. 210: $187-208$
Sánchez, J., M. Gil, L.H. Chasqui \& E.M. Alvarado. 2004. Grazing dynamics on a Caribbean reef-building coral. Coral Reefs 23: 578-583.

Santavy, D. \& E. Peters. 1997. Microbial Pests: coral diseases in the Western Atlantic. Proc. $8^{\text {th }}$ Int. Coral Reef Symp. 1: 607-612.

Schönberg, C.H.L. \& C.R. Wilkinson. 2001. Induced colonization of corals by a clionid bioeroding sponge. Coral Reefs. 20: 69-76.

Sutherland, K.P., J.W. Porter \& C. Torres. 2004. Disease and immunity in Caribbean and Indo-Pacific zooxanthellate corals. Mar. Ecol. Prog. Ser. 266: 273-302.

Szmant, A. 1991. Sexual reproduction by the Caribbean reef corals Montastrea annularis and M. cavernosa. Mar. Ecol. Prog. Ser. 74: 13-25.

Van Veghel, M.L.J. \& M. Kahman. 1994. Reproductive characteristics of the polymorphic Caribbean reef building coral Montastraea annularis. II. Fecundity and colony structure. Mar. Ecol. Prog. Ser. 109: 221-227.

Weil, E. 2004. Coral reef disease in the wider Caribbean: status and prognosis. p. 35-64. In E. Rosenberg \& Y. Loya (eds.). Coral disease and health. Springer, Berlin, Alemania.

Weil E. \& N. Knowlton. 1994. A multi-character analysis of the Caribbean coral Montastraea annularis (Ellis \& Solander, 1786) and its two sibling species, $M$. faveolata (Ellis \& Solander, 1786) and M. franksi (Gregory, 1895). Bull. Mar. Sci. 55: 151-175.

Weil, E., I. Urreiztieta \& J. Garzon-Ferreira. 2002. Geographic variability in the incidence of coral and octocoral diseases in the wider Caribbean. Proc. $9^{\text {th }}$ Int. Coral Reef Symp. Bali. 2: 1231-1237.

Williams, E.H., Jr. \& L. Bunkley-Williams. 1990. Coral reef bleaching alert. Nature 346: 225.

Zapata, P. 2005. Análisis histórico de los impactos naturales y antrópicos sobre los arrecifes coralinos del Parque Nacional Natural Corales del Rosario, Caribe Colombiano. Trabajo de Grado. Universidad Jorge Tadeo Lozano. Bogotá, Colombia.

Zea, S., J. Geister, J. Garzón-Ferreira \& J. Díaz. 1998. Biotic changes in the reef complex of San Andres Island (Southeastern Caribbean Sea, Colombia) occurring over nearly three decades. Atoll Res. Bull. 456: $1-30$. 